\title{
El Derecho a "que sea intentado" en Colombia a la luz del Estado Social de Derecho
}

\author{
The "Right to Try" in Colombia in Light of the Social \\ State of Law
}

\section{Daniela Agudelo Henao}

\author{
Abogada \\ Corporación Universitaria de Sabaneta - Colombia \\ Daniela_henao1105@hotmail.com
}

\author{
Yuliana Arenas Silva \\ Abogada \\ Corporación Universitaria de Sabaneta - Colombia \\ Yuli_arenas923@hotmail.com
}

\section{Resumen}

El derecho a "que sea intentado" o "right to try" fue reconocido mediante jurisprudencia de la Corte Constitucional a través de la sentencia T-057 de 2015, desde ese momento se inició un debate en torno a este nuevo derecho fundamental puesto que concierne dos puntos controversiales; por un lado, está el derecho a la vida en concordancia con la salud y la dignidad

\footnotetext{
Cómo citar este artículo:

Agudelo, D., y Arenas, Y. (2021). El Derecho a "Que Sea Intentado" en Colombia a la luz del Estado Social de Derechol. Revista de la Facultad de Derecho y Ciencias Políticas, 51(135). pp. 587-616. doi: https://doi.org/10.18566/rfdcp.v51n135.a13

Recibido: 26 de abril de 2020

Aprobado: 27 de mayo de 2021
} 
humana y, por otra parte, se encuentra el régimen de exclusión de la Ley Estatutaria de la Salud, donde se menciona que los procedimientos en fase de experimentación no serán financiados con los recursos del Estado.

Es por lo anterior que este nuevo derecho fundamental puede encontrar barreras en su aplicación por quienes lo requieren, ya que hasta el momento no se han establecido parámetros para su efectiva aplicación, generando un vacío jurídico respecto a esta nueva alternativa con la que cuentan los pacientes diagnosticados con enfermedades graves. Este texto tiene como propósito ilustrar a la comunidad académica y en general, sobre los alcances, definición y contenidos del derecho a "que sea intentado", por ser este un tema reciente e innovador que pone de presente tensiones entre principios y derechos constitucionales del Estado Social de Derecho.

\section{Palabras clave}

Derecho a que sea intentado; derecho a la salud; dignidad humana; procedimientos experimentales; right to try.

\section{Abstract}

The "Right to Try" was recognized through jurisprudence of the Constitutional Court through Sentence T-057 of 2015, since then, a debate began around this new fundamental right since it concerns two controversial points; on the one hand, there is the right to live in accordance with health and human dignity, while on the other hand, there is the exclusion regime of the Statutory Health Law, where it is mentioned that the procedures in the experimentation phase will not be financed with State resources.

Because of the above, this new fundamental right may find barriers in its application by those who require it since, so far, no parameters have been established for its effective application, generating a legal gap regarding this new alternative that patients diagnosed with serious diseases have. The purpose of this text is to illustrate to the academic community and in general, about the scope, definition, and content of the "Right to Try", as this is a recent and innovative issue that highlights the tensions between principles and constitutional rights of the State Social Law.

\section{Keywords}

right to try; right to health; human dignity; experimental procedures. 


\section{Introducción}

La Corte Constitucional ha reconocido una serie de nuevos derechos que no se encontraban explícitamente en la Constitución Política de 1991, pero que se desprenden de los principios constitucionales, entre estos nuevos derechos 0 derechos innominados se destaca el reciente derecho a "que sea intentado", el cual tiene el carácter de fundamental y, por su definición, sería inherente al ser humano.

El derecho a "que sea intentado", según la sentencia T-057 de 2015 que lo reconoce por primera vez:

guarda en sus orígenes una relación con el suministro de tratamientos, procedimientos y medicamentos experimentales para enfermos terminales, dado que se trata de un derecho inherente a la dignidad humana (art. 94 Superior), su ámbito de aplicación se extiende, mutatis mutandis, para el caso de los pacientes en estado vegetativo persistente (persistent vegetative state PVS) o de conciencia mínimo (minimally conscious state, MCS). (Corte Constitucional, 2015, p.35)

Aunque este derecho reconocido recientemente debería ser aplicado de manera directa ${ }^{1}$ pues se cataloga como un derecho fundamental, poseedor de dicha cualidad, existen algunas barreras para su efectiva realización, como lo es el régimen de exclusión consagrado en el artículo 15 de la Ley Estatutaria de la Salud 1751 de 2015, toda vez que, éste descarta a los tratamientos que se encuentran en fase de experimentación, de los procedimientos que serán financiados con los recursos del Estado.

Por otro lado, el bloque de constitucionalidad permitió que el derecho a la salud fuera reconocido bajo la categoría de derecho fundamental autónomo, a causa de que, contenía disposiciones, principios y valores materialmente constitucionales, a su vez, la jurisprudencia de la Corte Constitucional también generó gran impacto, debido a que, desde antes de la Ley Estatutaria -por ejemplo, en la sentencia T-760 de 2008- se reconoció dicho derecho bajo la categoría mencionada con anterioridad. Sin embargo, con la promulgación e implementación de la Ley 1751 de 2015 se positivizó y tomó mayor relevancia

1 Según el pronunciamiento de la Corte Constitucional en la sentencia T 406 de 1992, los derechos fundamentales son de aplicación inmediata por lo que no requiere la intermediación de la norma legal para que ellos tengan vigencia, por lo tanto, permiten la utilización inmediata de los elementos de protección de los derechos. 
dicha categoría; puesto que, esa Ley tuvo por objeto regular y establecer unos mecanismos de protección frente a este derecho, garantizando vía de ley la cobertura del sistema de salud a toda la población colombiana. Sin embargo, esta Ley estableció unos límites en cuanto a las prestaciones de salud garantizadas con recursos públicos, dejando esto una evidente afectación al derecho fundamental a "que sea intentado".

Es por lo anterior que, el presente artículo tiene como finalidad y objetivo principal analizar cuáles son las tensiones que se dan entre las restricciones a los métodos experimentales como componente del derecho a la salud y el derecho fundamental a “que sea intentado".

Para desarrollar lo anterior, el presente artículo se dividió en tres grandes momentos iniciando con la conceptualización de los derechos fundamentales, para de esta manera abordar el derecho fundamental a la salud y su evolución en el tiempo, en conexidad con el derecho fundamental innominado a "que sea intentado" o conocido en el common law como el "right to try".

Seguidamente, por el gran avance legislativo que ha tenido Estados Unidos respecto al derecho a "que sea intentado", se realizó un comparativo de la normatividad de este país con la colombiana, para esto se abordó desde su contexto histórico, ilustrando el caso de Abigail Burroughs por el impacto que este generó en Estados Unidos, llegando al punto de ser elemental para la historia del "Right to Try".

En tercer lugar, se analizaron las tensiones existentes entre el derecho fundamental a "que sea intentado" y el acceso a los procedimientos experimentales, por ser este último, una de las mayores limitaciones para el goce efectivo de este derecho.

Finalmente, el artículo concluye con las conclusiones respectivas desde un enfoque analítico.

La metodología que se empleó para cumplir con los objetivos planteados en el presente artículo fue cualitativa y de índole analítica, para esto se implementaron como métodos de recolección de información el análisis jurisprudencial respecto al derecho fundamental a la salud y los procedimientos experimentales. Así mismo, se realizó un estudio jurisprudencial con la finalidad de conocer lo establecido por la Corte Constitucional, quien es el órgano encargado de la integridad y la supremacía de la Constitución Política 
en relación con los temas anteriormente mencionados, por último, se efectuó un rastreo documental con el objeto de investigar los avances de estos temas.

Para esto se ejecutó una exploración y recolección de información encaminada al análisis del problema jurídico planteado, usando como criterios para la recopilación de estos, la pertinencia del documento, la relevancia para nuestro tema a profundizar y la confiabilidad de acuerdo con la fuente de información de donde este fue extraído.

Es por esta razón, que el presente artículo tiene como propósito ilustrar a la comunidad académica y en general, todo lo relacionado al tema de este nuevo derecho emergente, toda vez que es reciente e innovador, para esto, se desarrolló cada objetivo de manera independiente para llegar así a la conclusión del trabajo.

\section{Capítulo I: Ley Estatutaria 1751 de 2015 y el Derecho Fundamental a la Salud}

El Estado Constitucional en sentido fuerte surgió luego de las múltiples violaciones a los derechos humanos que tuvieron lugar durante la II Guerra Mundial. Este nuevo modelo de Estado buscaba reivindicar un criterio de validez material llamado Constitución, en el que además de una gama de derechos, se consagrarían unos mecanismos efectivos de protección, generalmente vía judicial. Así, hablar de estado constitucional en términos contemporáneos implica que todo el ordenamiento jurídico debe tener una coherencia o validez material, que todas las normas jurídicas se supeditan a la jerarquía máxima de la Constitución. (Amaya, 2015, p.90)

Colombia, al ser un Estado Constitucional de Derecho cuenta con una Constitución rígida y con supremacía constitucional, lo cual es una característica fundamental en este modelo, en esta se enmarcan una serie de reglas, principios y garantías mínimas que deben ser desarrolladas por todo el sistema jurídico para que, de esta manera, se pueda observar un efectivo derecho constitucional que irradie el ordenamiento jurídico.

Así mismo, en el ordenamiento jurídico se encuentran consagrados una serie de derechos los cuales deben ser protegidos y garantizados por este modelo de Estado. Es importante resaltar que, los derechos fundamentales 
son considerados como la estructura base de la sociedad y, es por esto, que se dota de mecanismos reales de protección de derechos, como lo son la acción de tutela, la acción popular, la acción de grupo, entre otros.

Por otro lado, el Estado Constitucional y los derechos fundamentales como elementos característicos de este modelo, vinculan a las tres ramas del poder con el objetivo de que se realice una efectiva división de este, donde la rama legislativa será la encargada de la elaboración y aprobación de leyes, la rama ejecutiva a su vez se encargará de gobernar y finalmente la rama judicial impartirá justicia aplicando debidamente el derecho.

Finalmente, es importante resaltar el papel del juez en el Estado Constitucional, toda vez que, este se encarga de la interpretación y creación de nuevos derechos y a su vez tiene el deber de realizar un control constitucional que en Colombia es mixto, pues lo realizan tanto los operadores jurídicos, como la Corte Constitucional quien es el órgano de cierre, lo anterior, se hace con el fin de salvaguardar los principios y garantías consagrados en la Carta Política, según Jorge Alejandro Amaya en el texto Control de Constitucionalidad. (Amaya, 2015, p.35)

\section{Contexto de los Derechos Fundamentales en Colombia}

Los derechos obedecen a circunstancias históricas, sin embargo, estos han evolucionado a través de los años, dado que, la sociedad no es estática y se hace necesario que el derecho evolucione en aras de garantizar derechos mínimos.

Para iniciar, es importante hacer una diferenciación entre los derechos fundamentales y los derechos humanos; los primeros son aquellos derechos mínimos que debe de garantizar cada Estado, se encuentren o no consagrados en la Constitución Política, mientras que los segundos, son todos los previstos en los tratados internacionales de derechos humanos. Según Monroy (2015), "la noción de "derechos fundamentales" proviene de la Constitución alemana de 1949, donde en su artículo I reconoce la importancia de estos derechos estableciendo que son inalienables al ser humano y que vinculan a las ramas del poder" (p.376)

Para la época en la que Alemania reconocía los derechos fundamentales, en Colombia regía la Constitución de 1886, este texto, consagraba como fuente suprema de toda autoridad a Dios, a la vez que otorgaba ciertas facultades al presidente de la República, pero dejaba por fuera los denominados derechos 
fundamentales. Esta Carta Política fue reformada en varias ocasiones, en 1910 se realizó una reforma liberal en la que se eliminaba la pena de muerte y se establecía que la elección de los presidentes se haría de manera directa.

Posteriormente, en 1936 se dio una nueva reforma donde se introdujeron algunos derechos sociales como la huelga, el trabajo, la función social de la propiedad, la reforma agraria, la asignación de tierras a campesinos, pero nuevamente el discurso de los derechos fundamentales quedaba excluido pues no contaban con un mecanismo efectivo para su protección.

Años más tarde, con la Asamblea Nacional Constituyente y la entrada en vigor de la Constitución Política de 1991, el concepto de derechos fundamentales llega a Colombia y se establece como uno de los pilares esenciales del Estado Social de Derecho, junto a los valores y principios constitucionales. Estos derechos en la Carta Política colombiana son una nueva categoría y se encuentran consagrados en la parte dogmática de la Constitución, pero no operan como una lista cerrada de derechos fundamentales. Es importante resaltar que, los derechos fundamentales no se van a definir de acuerdo con su ubicación en el texto constitucional, sino por la protección que brindan a la dignidad humana. (Martínez, 2016, p.123)

Este nuevo ordenamiento jurídico, inicialmente trajo consigo un listado en el que se establecían algunos derechos que serían considerados fundamentales expresamente, estos se encuentran consagrados en el Título II, Capítulo I, es importante resaltar que la Constitución Política no trajo una definición de estos, esta interpretación se ha obtenido a través del desarrollo jurisprudencial de la Corte Constitucional quien en virtud del artículo 241 de la Carta Política, es el Tribunal encargado de salvaguardar la Constitución y los derechos fundamentales en Colombia y quien los ha definido como "aquellos que (i) se relacionan funcionalmente con la realización de la dignidad humana, (ii) pueden traducirse o concentrarse en derechos subjetivos y (iii) encuentran consensos dogmáticos, jurisprudenciales o de derecho internacional, legal y reglamentario sobre su fundamentalidad" (Corte Constitucional, 2003).

Según lo anterior, los derechos fundamentales deben entenderse como derechos inalienables, ya que la esencia de estos reside en la dignidad humana, así mismo, según lo establecido por la Corte Constitucional, existe una conexión de estos con los principios constitucionales y tienen una eficacia directa, es decir, que no necesitan un desarrollo legal y son de aplicación inmediata, lo anterior se traduce en un derecho subjetivo que se entienden 
como competencias y facultades que se le otorgan a un ser humano, como afirma Monroy (2015, p. 379)

Corolario de lo precedente, se logra concluir que con la implementación de la Constitución Política de 1991 se generaron algunos avances significativos respecto a los derechos fundamentales, dado que, de conformidad con lo preceptuado en el artículo 94 constitucional, en Colombia se permiten garantizar derechos fundamentales que, sin encontrarse plasmados de manera expresa en la Constitución Política, son inherentes al ser humano, como es el caso del derecho a "que sea intentado", el cual es el tema central del presente artículo.

En ese orden de ideas, ya teniendo clara la noción de derecho fundamental, se hace necesario conocer la evolución que ha tenido el derecho a la salud en los últimos años, toda vez que a través de este, se permite la materialización de otros derechos. Así mismo, es relevante indagar sobre este tema en el entendido que tanto el derecho a la salud, como el derecho a "que sea intentado", tienen conexidad con otros derechos, tales como a la vida y a la dignidad humana, siendo este último uno de los principios fundamentales en un Estado Social de Derecho.

\section{El Derecho a la Salud en Colombia y su Evolución en el Tiempo}

Según el Preámbulo de la Constitución de la Organización Mundial de la Salud (2013) se entiende por salud "un estado de completo bienestar físico, mental y social, y no solamente la ausencia de afecciones o enfermedades”. Es por esto por lo que cada Estado debe trabajar para que su población goce del mejor estado de salud posible, además se necesita de este para que haya un desarrollo armónico de los demás derechos.

En razón de lo anterior, la salud en Colombia ha sido de constante debate ya que es un tema esencial y primordial para la sociedad, debido a esto, el Estado ha tenido la necesidad de implementar medidas para su mejoría y a su vez garantizarla como un derecho, dado que inicialmente la Constitución Política lo consagraba como un servicio público esencial a cargo del Estado. Para profundizar lo expuesto, se resalta que el Estado colombiano, ratificó a través de la Ley 74 de 1968 el Pacto Internacional de Derechos Económicos, Sociales y Culturales, adoptado por la Asamblea General de las Naciones Unidas, acogiéndose en su artículo 12, el cual establece que, "Los Estados 
Partes en el presente Pacto reconocen el derecho de toda persona al disfrute del más alto nivel posible de salud física y mental.” (p. 5)

Como se mencionó precedentemente, uno de los objetivos de la Carta Política de 1991 fue dotar al pueblo colombiano de derechos fundamentales; sin embargo, inicialmente parecía que dejó el derecho a la salud por fuera de esa categoría, pues contempló a este en el artículo 49 como un servicio público bajo la dirección, coordinación y control del Estado, esto generó múltiples dificultades en cuanto al acceso de la prestación de servicios, debido a que el Estado no contaba con la suficiente capacidad para prestar un servicio real y eficiente.

En consecuencia de lo anterior, se creó la Ley 100 de 1993 con la finalidad de prestar servicios de salud en calidades dignas a través de las Entidades Promotoras de Salud -EPS- y las Instituciones Prestadoras de Servicios de Salud -IPS-, sin embargo, nuevamente se presentaron barreras teniendo en cuenta que una gran cantidad de personas no podía acceder a tratamientos médicos ya que se presentaban límites económicos, esto generó en la sociedad colombiana gran preocupación y la necesidad de tomar acciones oportunas e inmediatas, puesto que, los derechos fundamentales no pueden depender de factores económicos. (Jiménez et al. 2016).

Con fundamento en lo precedente, en el año 2012 el juez constitucional comienza a reconocer y a destacar con mayor relevancia el valor de la dignidad humana, al exigir una protección permanente, de igual manera, se empieza a definir la naturaleza jurídica de los derechos económicos, sociales y culturales. Un claro ejemplo de esto es a través de la sentencia T-406 de 1992, donde el magistrado ponente Ciro Angarita Barón, argumenta que en ocasiones los derechos económicos, sociales y culturales pueden ser considerados como fundamentales siempre y cuando sea indudable su conexidad con un principio o derecho fundamental. Debido a esto, dichos derechos son reivindicables a través de la acción de tutela considerando que, según la Corte Constitucional, son “derechos fundamentales por conexidad”. (Corte Constitucional, 1992)

En virtud de lo expuesto, en el año 1995 la Corte Constitucional por medio de la sentencia T-207, estableció nuevamente que, al momento de negar una atención médica, sin importar su razón, se afectaba la dignidad humana, por lo que se podía recurrir a la acción de tutela. (Corte Constitucional, 1995)

No obstante, dicho pronunciamiento no fue suficiente para generar el impacto que buscaba el derecho a la salud; por lo tanto, a través de la sentencia 
de unificación jurisprudencial SU-111 de 1997, se determinó que el derecho a la salud solo era fundamental cuando se establecía conexidad ${ }^{2}$ entre el derecho a la vida, el servicio de salud y la dignidad humana. Sin embargo, nuevamente se causó una afectación preocupante, pues primero se tenía que evidenciar un grave deterioro en la salud para poder así garantizar la dignidad humana.

Contrario a lo anterior, algo muy diferente ocurría con los niños, niñas y adolescentes pues según el artículo 44 de la Constitución Política el derecho a la salud es catalogado para ellos como un derecho fundamental, y así mismo sucedía con los demás sujetos de especial protección constitucional ${ }^{3}$ a quienes el Estado sí les garantizaba el derecho a la salud como un derecho fundamental autónomo sin necesidad de establecer relación de conexidad puesto que sus derechos son de aplicación inmediata por su situación de debilidad manifiesta e indefensión.

No fue sino hasta el año 2008 que a través de la sentencia T-760 se pudo establecer el derecho a la salud para todos los habitantes de Colombia como un derecho fundamental autónomo por tratarse de una necesidad básica humana, sin embargo, se pudo constatar con lo anterior el amplio recorrido que tuvo que acudir este derecho para que se reconociera realmente por su inherencia a la persona, sin distinción alguna.

Es menester resaltar que, la sentencia aludida con antelación fue trascendental en el cambio del Sistema de Salud en Colombia, dado que, en esta, la Corte Constitucional analizó un conjunto de casos en los que se vulneraba el derecho a la salud, como consecuencia de múltiples factores, dejando en evidencia la necesidad de una restructuración del sistema.

En consecuencia de lo anterior en esta sentencia, entre otras cosas, se resaltó la obligación que tiene el Estado de establecer las reglas necesarias para que las EPS y las IPS puedan garantizar de manera plena la prestación de los servicios que sean requeridos por las personas y, de esta manera, eliminar los obstáculos irrazonables que impedían la materialización del derecho a la

2 Según lo expuesto por la Corte Constitucional en la sentencia T-491 de 1992, los derechos fundamentales por conexidad son aquellos que a pesar de no ser denominados como fundamentales en la Constitución Política, se les clasifica de esta manera en virtud de la íntima e inescindible relación con otros derechos fundamentales.

3 De acuerdo con lo expuesto por la sentencia T 881 de 2008, son sujetos de especial protección constitucional los siguientes: (i) los menores, (ii) las mujeres en estado de embarazo o madres cabeza de familia, (iii) los adultos mayores, (iv) los discapacitados físicos y mentales, (v) los indígenas y las minorías étnicas, (vi) las minorías sexuales, (vii) las personas en estado de indigencia y (viii) las personas en situación de desplazamiento. 
salud a un sector de la población. En esa misma línea, la Corte señaló que todas las personas tienen derecho a que se les garantice el acceso a todos los servicios de salud que este requiera dado que, de esta manera, se asegura una mayor efectividad de este derecho.

Así las cosas, luego de que la Corte Constitucional efectuará un arduo análisis de las problemáticas que impedían que el derecho a la salud fuera garantizado de manera plena, en esta sentencia se impartieron diversas órdenes encaminadas a reestructurar el Sistema de Salud, lo anterior con el objetivo de establecer que el derecho a la salud tiene el carácter de ser fundamental para toda la población, en este punto, es importante advertir que si bien el juez le da el status de derecho fundamental, no será sino hasta la promulgación de la Ley 1751 de 2015 que esto queda positivizado.

Así mismo se hace necesario resaltar que, con la finalidad de solucionar las diferencias de la Ley 100 de 1993, la Corte Constitucional ordenó al Ministerio de Protección Social adoptar las medidas necesarias para asegurar la cobertura universal sostenible del Sistema General de Seguridad Social en Salud, pues solo así, podría garantizarse este derecho fundamental a toda la población.

Finalmente, fue tomando en cuenta toda la trayectoria jurisprudencial mencionada anteriormente que nació la necesidad de implementar una ley para lograr la efectividad del derecho fundamental a la salud para todos los habitantes, y a su vez establecer sus mecanismos de protección y aclarar de qué se trata de un derecho autónomo e irrenunciable, no obstante, no fue sino hasta el año 2015 que se logró garantizar este derecho a través de la Ley Estatutaria 1751. Es menester resaltar que esta Ley cumple con todas las órdenes impartidas por la Corte en la sentencia T-760 de 2008, en la Observación 14 y en los instrumentos internacionales en aras de garantizar este derecho sin distinción alguna.

\section{Surgimiento del Derecho Fundamental a "Que Sea Intentado" en Colombia}

La jurisprudencia ha sido reconocida históricamente como fuente auxiliar del derecho, es decir, esta solo era aplicable cuando la Ley que era considerada la fuente primaria de derecho guardara silencio sobre un tema específico; es por esto que anteriormente los jueces se encargaban de aplicar mecánicamente la ley sin crearla o modificarla, ya que el paradigma positivista 
de interpretación sostenía que el juez era la boca de la ley, y solo tenía que aplicarla sin interpretarla (Aguiló, 2007 p. 3).

Con el paso de los años y con el auge del neoconstitucionalismo como modelo interpretativo, se le atribuyó gran importancia a la Constitución, toda vez que se reconoció que esta se encontraba sobre las demás normas jurídicas. Así mismo, este nuevo modelo, se fundamentó en la división de poderes y en la defensa de los derechos.

Por otro lado, como se mencionó precedentemente, la jurisprudencia tomó un papel relevante, máxime si se trata de jurisprudencia constitucional que tiene un carácter vinculante; por esta razón, tras la Constitución de 1991, Colombia adoptó un sistema de precedentes fuerte, en el que el papel del juez es fundamental, pues se considera que, actualmente "los derechos son aquellos que los jueces dicen a través de las sentencias de tutela” (Corte Constitucional, 1992)

La Constitución como base del ordenamiento jurídico debe ser asegurada, por esta razón se emplean modelos de control constitucional, los cuales velan por la efectiva primacía de la Carta Política frente a las demás normas. Como se mencionó anteriormente, en Colombia, se emplea un tipo de control mixto, toda vez que es difuso y concentrado.

Es considerado difuso en el entendido que, según el artículo 4 constitucional, todos los jueces tienen la competencia de dejar de aplicar una ley u otra norma por ir en contravía de la Constitución, además todos los jueces en Colombia conocen de acciones de tutela; en otras palabras, están facultados para pronunciarse en casos específicos sobre la validez de una norma respecto de la Constitución.

Porotrolado, la CorteConstitucional emplea unmedio decontrol concentrado, puesto que, según la Carta Política de 1991, es el Tribunal encargado de salvaguardar la supremacía de la Constitución (art. 241 constitucional) y, por ende, es este el encargado de la acción pública de inconstitucionalidad frente a leyes y otras normas que contraríen el ordenamiento jurídico, además de esto, se encargan de la revisión de tutelas para unificar criterios con las sentencias denominadas T y SU.

Como se ha manifestado precedentemente, la Constitución Política de 1991 trajo un catálogo de derechos que deben tener los habitantes del territorio colombiano, es importante aclarar que esto no es un impedimento para 
que se pueda dar la creación de nuevos derechos, ya que, el artículo 94 del Texto Constitucional, menciona que los que se encuentran consagrados en este precepto, no deben ser entendidos como negación de otros que no se encuentran consagrados de manera expresa.

Para la determinación de estos nuevos derechos la Corte Constitucional estableció unos criterios para delimitar cuáles pueden ser considerados fundamentales y, por consiguiente, susceptibles de amparo vía acción de tutela, y mencionó que son fundamentales aquellos que tienen un carácter inherente respecto al núcleo jurídico, social, político y cultural del hombre. A este criterio puede llamársele material, de acuerdo con Tulio Chinchilla. Según el criterio material los derechos fundamentales se identifican por su naturaleza misma, puesto que poseen una cualidad valorativa que lo determina así; es decir, se supera el mero criterio formal y se establece que un derecho es fundamental cuando tenga conexión con un principio constitucional, y finalmente son derechos fundamentales aquellos que tengan una eficacia directa $\mathrm{y}$ un contenido esencial, esto último según los criterios auxiliares, complementarios o técnicos. (Chinchilla, 2009, p.32),

Es teniendo en cuenta todo lo anteriormente mencionado que la Corte Constitucional colombiana, con el fin de proteger la Constitución y garantizar la dignidad humana de todos los habitantes del territorio, reconoció recientemente a través de la sentencia T-057 de 2015 el derecho fundamental a "que sea intentado", el cual, a pesar de no estar en la Carta Política, fue reconocido y protegido por mecanismos constitucionales dado su conexidad con el derecho a la vida, la dignidad humana y la salud.

En este punto, es importante advertir que el derecho a la vida se cataloga como el primer derecho de la persona humana, este, no se reduce únicamente a la existencia biológica, sino que también se refiere a la posibilidad de desarrollar un determinado proyecto de vida. En ese orden de ideas, se deja en evidencia la trascendencia del derecho a "que sea intentado" dado que, a través de este, se busca que la persona pueda autodeterminarse y tomar las decisiones que considere sean las pertinentes para su vida sin que el Estado y la sociedad en general interfieran en esto. Así mismo, es trascendental tener en cuenta que, como lo señaló la Corte Constitucional, “asegurar la vida, no es solo el derecho subjetivo que se tiene sobre la vida, sino la obligación de los otros a respetar el derecho a seguir viviendo o a que no se anticipe la muerte" (Corte Constitucional, 1996). Es por lo anterior, que la Corte considera necesario, reconocer este nuevo derecho pues, de no hacerse, se estaría en 
primer lugar, interfiriendo en el plan de vida de las personas que padecen una enfermedad grave, pero que tienen el deseo de continuar viviendo, y en segundo lugar, se estaría anticipando la muerte de aquellos quienes padecen enfermedades que no cuentan con un tratamiento comprobado, sino que, este se encuentra en la fase de experimentación.

Corolario de lo anterior, en este desarrollo jurisprudencial la Corte analizó el siguiente caso: el padre de la joven Mairoby Rivera quien fue diagnosticada en "estado vegetativo persistente" 4 - después de sufrir un accidente de tránsito - presentó acción de tutela contra la EPS Sanitas y Colsanitas Medicina Prepagada para que le suministraran la debida atención a su hija. El 30 de noviembre del año 2009, el Juzgado 28 Penal de Medellín, ordenó a la EPS antes mencionada brindarle atención integral a la paciente.

Años más tarde, el médico que venía tratando a la joven Mairoby le recomendó realizarse el tratamiento "Estimulación espinal epidural cervical”, pues posiblemente era la única alternativa para mejorar la calidad de vida de la paciente, por lo que el padre realizó la respectiva solicitud, pero ante esta la EPS se negó, argumentando que no funcionaría y que el tiempo para realizar este tratamiento ya había pasado.

Fue por esa respuesta que el padre de la joven, actuando como agente oficioso, presentó incidente de desacato, frente al fallo proferido el día 30 de noviembre de 2009. Mediante auto del 27 de noviembre de 2013, el juzgado se abstuvo de iniciar el trámite de desacato argumentando que el tratamiento se encontraba fuera del POS, por tratarse de un procedimiento experimental.

Es oportuno resaltar que la posición que adoptó la Corte giró en torno a que, aunque el tratamiento ordenado por el neurocirujano funcional era experimental, este habría mostrado algunos avances positivos frente a

4 Según la Sentencia T 057 de 2015, el estado vegetativo persistente trata de personas que mantienen sus funciones cardiovasculares, respiratorias, renales, termorreguladoras y endocrinas, así como la alternancia sueño-vigilia, pero que no muestran ningún tipo de contacto con el medio externo y ninguna actividad voluntaria.

5 La estimulación cerebral profunda es un procedimiento que se viene realizando desde hace 25 años, y ha demostrado ser efectivo en determinadas patologías, en la actualidad se encuentra aprobada para el tratamiento del Parkinson, temblor, distonía y trastorno obsesivo compulsivo, para estas patologías existe suficiente evidencia sobre su efectividad y seguridad y está aprobado su uso por los diferentes estamentos internacionales, en el caso de los Estados Unidos, por la FDA. 
una posible solución a esta patología, por lo que este tratamiento era una oportunidad que tenía la paciente de mejorar su salud. ${ }^{6}$

Es por lo anterior que la Corte Constitucional luego de un arduo debate, reconoció la existencia del derecho innominado a "que sea intentado" en aquellos pacientes que se encuentren en estado vegetativo persistente o de conciencia mínima, para esto examinó los recientes desarrollos legislativos y los fallos que se han presentado en algunos estados de la Unión Americana y así de esta manera, la Corte Constitucional (2015) definió este nuevo derecho como:

El derecho que tiene un paciente diagnosticado en estado vegetativo persistente o de mínima conciencia, de agotar todas las posibilidades científicas existentes, sin importar el carácter experimental que este tenga, puesto que lo que se busca es preservar o mejorar la calidad de vida de quien padece la enfermedad y correlativamente garantizar la dignidad humana que es inherente a la persona. (Corte Constitucional, 2015)

Finalmente, en esta sentencia, la Corte Constitucional revocó los fallos proferidos anteriormente donde, en primera instancia, el Juzgado 23 Penal Municipal de Medellín declaró improcedente el amparo solicitado por el señor Mario de Jesús Rivera, quien estaba actuando en representación de su hija, argumentando que, se configuraba la cosa juzgada constitucional, por existir identidad en los sujetos, los derechos reconocidos y las pretensiones; a su vez, el Juzgado Sexto Penal del Circuito de Medellín en segunda instancia, confirmó la sentencia de primera instancia, estimando que, lo pretendido se basaba en los mismos supuestos fácticos de lo solicitado en la primera acción de tutela.

Por consiguiente, a través de la sentencia T-057 de 2015 se amparó el derecho a "que sea intentado" y se ordenó a la EPS Sanitas y Colsanitas Medicina Prepagada a que, en un término no superior a las 48 horas siguientes, autorizara la realización del tratamiento de "estimulación espinal epidural para el tratamiento de estados de subconsciencia y programación de voltajes adecuados para el caso".

6 En torno al caso de Mairoby surgieron múltiples posiciones respecto de varios especialistas en el tema, toda vez que, mientras el Dr. Páez Novoa manifestaba que el tratamiento era la única alternativa que tenía la paciente de mejorar su calidad de vida, y exponía detalladamente el procedimiento de la cirugía, la cual no consideraba experimental, ya que, se le había realizado a varios pacientes y había demostrado su capacidad frente a varias patologías, el médico de la EPS expresaba que las posibilidades de que la paciente mostrara mejoría al someterse a este procedimiento eran nulas, además resaltaba que, este tratamiento había tenido resultados negativos en personas en estado vegetativo, por lo que no debía realizarse. 
Es importante señalar que el fallo de esta sentencia no fue unánime, ya que el Magistrado Luis Ernesto Vargas Silva presentó salvamento de voto argumentando que se podrían generar graves consecuencias al darle el carácter de fundamental al derecho a intentar, más aún cuando un grupo de expertos expusieron los riesgos que se podían presentar al realizar el procedimiento ordenado por el neurocirujano que venía tratando a la paciente.

En último lugar, argumentó que en la Sentencia no se había realizado el ejercicio de valoración probatorio que se exigía por la complejidad del caso, sino que se basó únicamente en la traducción libre de los artículos académicos aportados por el médico tratante. Lo que denota que en el seno de esta decisión hubo un debate técnico especializado, generalmente ajeno a la labor de los jueces.

\section{Capítulo II: El derecho fundamental a "que sea intentado" según La Corte Constitucional y la legislación norteamericana}

\section{El "Right to Try" en Estados Unidos}

Con la finalidad de regular la conducta de los habitantes de su territorio, fijar reglas en la sociedad y definir su estructura estatal, cada país tiene la potestad y autonomía de implementar su propio sistema jurídico, integrado a través de la Constitución Política, leyes y jurisprudencia.

Desde sus comienzos, Estados Unidos estuvo influenciado por el periodo colonial inglés, es decir, el derecho inglés o common law, no obstante, esto se dio por terminado a partir de la independencia de las trece colonias y la implementación de la Constitución Federal de 1788.

Con lo anterior, Estados Unidos comenzó a transformarse en un sistema jurídico combinado entre los precedentes judiciales y la legislación, toda vez que, ambos se necesitan para la toma de decisiones judiciales. Considerando lo anterior, se abordará el "Right to Try" en Estados Unidos.

El derecho a "que sea intentado" o mejor nominado "Right to Try" debido a su surgimiento en Estados Unidos de América, tiene como finalidad que los pacientes con enfermedades terminales tengan la posibilidad de acceder a tratamientos experimentales o medicamentos que hayan alcanzado la primera 
fase de la Administración de Medicamentos y Alimentos de los Estados Unidos de América (FDA por sus siglas en inglés) ${ }^{7}$, actualmente, este mecanismo es fácil de acceder en dicho país por su regulación; sin embargo, esto ha sido gracias a la constante lucha de varias organizaciones e individuos, como es el caso de Abigail Burroughs.

Abigail Burroughs fue una gran impulsora de este derecho, pues con tan solo 19 años, fue diagnosticada con cáncer en su cuello y cabeza, por esta razón intentó a través de varios tratamientos médicos mejorar su estado de salud; no obstante, ninguno generó efecto en ella, debido a esto, sus médicos le recomendaron iniciar un tratamiento posiblemente eficaz denominado Erbitux ${ }^{8}$, sin embargo, este no se pudo realizar puesto que no estaba aprobado por la Administración de Medicamentos y Alimentos de los Estados Unidos de América -FDA-.

Lo anterior causó en ella y en su familia gran desesperación, debido a que era su última posibilidad y esperanza médica, incluso, lucharon durante los últimos 7 meses por su acceso. Lamentablemente el 9 de junio de 2001, Abigail falleció a sus 21 años sin la oportunidad de saber si ese tratamiento hubiera efectivamente mejorado su diagnóstico.

Fue debido a estos hechos, que su padre, Frank Burroughs, impulsó la Alianza Abigail (The Abigail Alliance for Better Access to Developmental Drugs) en todos los medios de comunicación; incluso, llegando a las salas de justicia ya que él buscaba impulsar que la Administración de Medicamentos y Alimentos de los Estados Unidos de América -FDA- le permitiera a los pacientes en estado terminal acceder a tratamientos experimentales y medicamentos, pues él consideraba que esto era un derecho constitucionalmente protegido.

Con lo anterior, a pesar de existir varias organizaciones y sujetos en favor del "Right to Try", según un artículo publicado por CNN el 29 de mayo de 2018 , existen algunos oponentes, como es el doctor Steve Joffe que argumenta que el

7 Según lo descrito por la FDA, su función consiste en: proteger la salud pública mediante la regulación de los medicamentos de uso humano y veterinario, vacunas y otros productos biológicos, dispositivos médicos, el abastecimiento de alimentos en nuestro país, los cosméticos, los suplementos dietéticos y los productos que emiten radiaciones. Favorecer la salud pública mediante el fomento de las innovaciones de productos. Proveer al público la información necesaria, exacta, con base científica, que le permita utilizar medicamentos y alimentos para mejorar su salud.

8 El Instituto Nacional del Cáncer de los Institutos Nacionales de la Salud de EEUU define el Erbitux como un medicamento que se usa para tratar ciertos tipos de cáncer de cabeza y cuello, y cierto tipo de cáncer colorrectal que se diseminó hacia otras partes del cuerpo. 
derecho a "que sea intentado” podría generar un perjuicio en la salud pública, puesto que, los medicamentos serian facilitados con poco conocimiento acerca de sus riesgos y beneficios, así mismo, ya existía la posibilidad de aplicar y participar en ensayos clínicos. (Howard, 2018).

\section{El "Right To Try" en Estados Unidos y la Ley Federal S.204}

Con lo mencionado anteriormente, el "Right to Try" desde el año 2001 empezó a regularse significativamente en 41 Estados, pues consideraban que era un derecho fundamental del paciente intentar mejorar su estado de salud bajo todas las circunstancias posibles, por esto, según un informe realizado en el año 2016 por el diario científico Therapeutic Innovation \& Regulatory Science, desde enero del 2005 a diciembre del 2014, la FDA ha recibido 8,922 solicitudes para acceder a medicamentos y tratamientos experimentales, siendo aprobados un 99.3\% de estas (Jarow et al, 2016).

No obstante, no fue sino hasta el 30 de mayo de 2018 que el presidente Donald Trump, ratificó la Ley Federal S.204, generando que el "Right to Try" se convirtiera en una ley uniforme para los 50 Estados de Norteamérica y, a su vez, se establecieron ciertos criterios en cuanto a quiénes pueden solicitar y qué medicamentos y tratamientos clasifican. Entre ellos se resaltan dos requisitos: que el paciente debe ser diagnosticado con una enfermedad o afección potencialmente mortal y que haya agotado todas las opciones de tratamiento aprobadas; de igual manera, debe presentar su consentimiento de manera escrita donde se pronuncie con respecto a los posibles riesgos asociados con su procedimiento. En cuanto a los medicamentos y tratamientos experimentales, se hace indispensable que estos hayan aprobado la primera fase $^{9}$ de la FDA y estar en un ensayo clínico activo.

En conclusión, el "Right to Try” ha influenciado en la legislación colombiana con el propósito de generar esperanza y una oportunidad más de vivir, ya que este busca que los pacientes en estado crítico de salud puedan tener la libertad de elegir un tratamiento así esté en fase experimental.

9 La primera fase consiste en evaluar por primera vez un medicamento o tratamiento experimental en un grupo reducido de entre 20 a 100 personas, los investigadores evalúan la seguridad del tratamiento, determinan un intervalo de dosificación seguro e identifican los efectos secundarios. 


\section{Diferencias del Derecho a "Que Sea Intentado" en Colombia y Estados Unidos de América}

En la actualidad, el derecho comparado se ha convertido en una herramienta importante para analizar minuciosamente el desarrollo normativo que han presentado las sociedades del mundo entero respecto a determinados temas que anteriormente eran considerados indiscutibles, pero, que actualmente con la evolución de la sociedad, deben ser evaluados de una manera más razonable y justa, para de esta manera permitir una armonización del derecho en general (Corte Constitucional, 2006).

En relación con Colombia y Estados Unidos de América, se logran evidenciar de manera clara grandes diferencias en su ordenamiento jurídico, entre ellas se puede resaltar que la Constitución Política de los EEUU se caracteriza por encontrarse escrita en términos amplios, es decir, establece de manera general la forma de gobierno, el cual se caracteriza por ser federal; es por esto que cada Estado tiene una Constitución autónoma en la que se establecen de manera detallada los parámetros aplicables dentro de un territorio determinado y, por ende, son Estados soberanos.

Es importante resaltar que una de las principales fuentes del derecho inglés es el derecho común o también denominado common law, este se basa en las decisiones adoptadas por los Tribunales en casos específicos, los cuales crearon un precedente normativo que deberá ser adoptado por los demás jueces cuando se presenten casos análogos, con esto se busca que las decisiones de los jueces sean más estables y predecibles.

Debe destacarse que, por la forma de gobierno de los Estados Unidos, no existen los mismos derechos para todos sus habitantes, toda vez que, cada Estado tiene la potestad de crear nuevos derechos que sólo regirán en un territorio específico, como es el caso del derecho a "que sea intentado" o "Right to Try".

El "Right to Try" como se evidenció en acápites anteriores, se encuentra ampliamente regulado en los Estados Unidos, por esto en los últimos años Colombia, a través de la Corte Constitucional como Tribunal encargado de garantizar la efectividad de los derechos constitucionales, ha adoptado directrices de la regulación americana respecto del "Right to Try" para constituir este derecho en el país, para ello se ha basado en la regulación de 5 Estados que habían establecido parámetros para la aplicación de este derecho. 
Si bien la Corte Constitucional reconoció este nuevo derecho, los procedimientos experimentales no pueden ser aplicados en ninguna circunstancia, puesto que se encuentran dentro de la lista de exclusión de la Ley Estatutaria de la Salud, por ende, no serán financiados con los recursos del Estado, con esto se deja en evidencia una de las más sólidas barreras que presentan los pacientes al momento de solicitar la efectividad del derecho a que sea intentado. Corolario de lo anterior, es claro que se presenta una tensión entre este nuevo derecho y la sostenibilidad financiera del Sistema de Salud, dado que actualmente no se cuentan con los recursos requeridos para suministrar el personal médico y las herramientas necesarias para implementar tratamientos en fase de experimentación, esto sumado a la deuda histórica que ha permeado el Sector de la Salud desde hace varios años.

No obstante, en Colombia se siguen suscitando algunos debates frente a este derecho, puesto que este presenta numerosos vacíos al no estar legislado, lo que genera que no pueda ser aplicado de manera directa y por ende que no sea una alternativa viable para los pacientes que presenten enfermedades terminales. Cabe advertir que, el derecho a "que sea intentado", requiere de una participación de toda la sociedad en los aspectos ético, social, cultural y jurídico.

\section{Capítulo III: Tensiones Entre el Derecho a "Que Sea Intentado" y la Regulación del Acceso a los Procedimientos Experimentales}

\section{Procedimientos Experimentales}

Con la transformación de la sociedad y el avance de la tecnología, se han ido presentando nuevos escenarios respecto al tema de la medicina, entre estos se destaca el derecho a "que sea intentado"; este nuevo derecho suscita muchos debates, por englobar temáticas tan controversiales como la ética y el sometimiento voluntario de personas a tratamientos que aún se encuentran en fase de experimentación, únicamente con el objetivo de prolongar su vida. Por esta razón, antes de abordar los procedimientos experimentales es necesario inicialmente hablar de bioética.

La bioética es entendida como la búsqueda de la ética adaptada a los temas planteados por el progreso de la sociedad; esta, tiene como pilar esencial brindar soluciones a los problemas que se presenten en las ciencias de la 
vida respecto de la ética, así mismo, es considerada como un movimiento interdisciplinario, pues se encuentra relacionado con la filosofía y el derecho. (Escobar Cosme, 2010)

La primera, se encarga de brindar una orientación frente a las decisiones adoptadas por la ética, mientras que la segunda se encarga de legislar sobre los temas que son controvertidos, aunque no siempre estos problemas son ampliamente regulados, como es el caso de los procedimientos experimentales. (Mazo, 2014, p.2)

En Colombia, los procedimientos experimentales carecen de regulación; según lo consagrado en el artículo 15 de la Ley Estatutaria de la Salud 1751 de 2015, estos no son financiados con los recursos del Estado pues son procedimientos que todavía no se encuentran acreditados y que, por ende, no se tiene una certeza sobre las consecuencias que pueda generar en el cuerpo humano y a su vez, son de un costo - beneficio elevado (Corte Constitucional, 2013). Para sustentar lo aludido, a través de la página web del Ministerio de Salud y Protección Social de Colombia se pueden consultar los actos administrativos desde el año 2017 al 2020, donde establecen el listado de servicios y tecnologías que serán excluidas de la financiación con recursos públicos asignados al sector de la salud ${ }^{10}$.

La Corte Constitucional ha sostenido en reiterada jurisprudencia que, para que un tratamiento no sea considerado experimental, es decir, sea una alternativa terapéutica válida, se debe demostrar su idoneidad como tratamiento frente a una enfermedad, esto se hará a través de una acreditación tanto de la comunidad científica, como de entidades especializadas en acreditación. En este proceso se busca analizar detalladamente los resultados de un tratamiento, con el fin de establecer con claridad los efectos tanto positivos como negativos que podrían generar en los pacientes para, de esta manera, garantizar sólo aquellos que demuestran mayor efectividad. (Corte Constitucional, 2013)

No obstante, la Corte Constitucional ha establecido algunos criterios para que se pueda ordenar el suministro de procedimientos, exámenes o medicamentos que no se encuentran en el POS -actualmente Plan de Beneficios en Salud-, estos son:

10 Año 2017: Resolución 5267 de 2017 y Resolución 330 de 2017. Año 2018: Resolución 687 de 2018. Año 2019: Resolución 244 de 2019. Año 2020: Resolución 965 de 2020. 
(i) Que la falta del procedimiento excluido, amenace los derechos fundamentales de la vida o la integridad personal del paciente; (ii) Que se trate de un procedimiento que no pueda ser sustituido por uno de los contemplados en el POS o que, pudiendo sustituirse, no obtengan el mismo nivel de efectividad que el excluido del plan; (iii) Que el servicio médico haya sido ordenado por un médico adscrito a la entidad encargada de garantizar la prestación de servicios a quien está solicitándolo, y (iv) Que el paciente realmente no pueda sufragar el costo del medicamento o tratamiento requerido, y que no pueda acceder a él por ningún otro modo o sistema. (Corte Constitucional, 2013)

Respecto de los procedimientos experimentales, se logra observar que en la mayoría de los casos estos tienen un vínculo estrecho con el derecho fundamental a la salud, toda vez que, si bien el Estado debe garantizar la salud en condiciones dignas, y por ende se considera que solo debe financiar tratamientos que se encuentren autorizados y de los cuales se tenga certeza sobre su efectividad, en algunos casos los tratamientos que aún se encuentran en fase de experimentación pueden ser la única alternativa para los pacientes que se encuentran diagnosticados con enfermedades graves, por lo que estos procedimientos no deberían estar excluidos del Sistema de Salud, por el contrario, deberían realizarse, priorizando la voluntad del paciente, ya que este es considerado como un sujeto moral capaz de tomar sus propias decisiones.

La Corte Constitucional ha tocado de manera tangencial el tema de los procedimientos experimentales, como se evidencia a continuación en la siguiente jurisprudencia; la sentencia SU-337 de 1999, se enfoca principalmente en el consentimiento informado del paciente al momento de someterse a una intervención experimental, el cual debe ser libre de engaños, para de esta manera no cosificar a la persona y convertirla en un instrumento, sino para darle su estatus de sujeto coparticipe del desarrollo de la ciencia. Así mismo, la sentencia T-216 del 2008, resalta la importancia del consentimiento del paciente, ya que este va enlazado con el principio de dignidad y autodeterminación.

A su vez, la sentencia T-310 de 2013, señala que los medicamentos excluidos del Plan Obligatorio de Salud hoy - Plan de Beneficios en Salud-, se pueden reclamar vía acción de tutela, para esto, se debe demostrar que este medicamento es el único que puede surtir efectos favorables en el paciente. Finalmente, la sentencia T-1330 de 2005, señala que: 
la prohibición absoluta del financiamiento de actividades, intervenciones, procedimientos y medicamentos experimentales con recursos provenientes del sistema puede resultar desproporcionada y por lo tanto vulneradora del derecho a la salud, bien sea como un derecho fundamental autónomo o conexo, no sólo cuando esté en riesgo la vida del peticionario sino también cuando existan reales posibilidades de recuperación o de mejoría. (Corte Constitucional, 2005, p. 12)

No obstante a lo anterior, se hace necesario que el Congreso de la República como máximo órgano representativo del poder legislativo, legisle sobre el tema y establezca unos parámetros aceptables, esto es, que señale unos requisitos mínimos para que se pueda emplear una efectiva realización de estos tratamientos sin necesidad de acudir a la acción de tutela, para que de esta manera el derecho positivo cumpla con el orden establecido para la producción de normas que regirán al país, las cuales deben ser la voluntad del pueblo y deben ser coherentes con la Constitución, pues este es el parámetro de validez de las demás normas.

Así mismo, se hace necesario que existan leyes sobre el tema en las cuales se establezcan los requisitos para acceder a procedimientos experimentales, la edad mínima para manifestar el consentimiento de querer someterse a estos tratamientos y la forma en la que se debe manifestar; establecer en los casos de que la persona no pueda manifestar su voluntad, debido a que padezca de alguna enfermedad que se lo impida, quien podría hacerlo por el paciente y finalmente, con qué recursos se van a financiar estos procedimientos.

A pesar de que en los últimos años se ha reconocido el rol de los jueces como creadores de derecho, el precedente judicial no se constituye como una garantía para que todos los jueces fallen de la misma manera, puesto estos se pueden apartar de la línea jurisprudencial trazada, siempre y cuando justifiquen adecuadamente su decisión, aunque se considere que esto es violatorio del principio de igualdad

\section{Barreras de Acceso a la Salud en los Procedimientos Experimentales}

Una de las principales características del Sistema de Seguridad Social en Salud, con la creación de la Ley 100 de 1993, es que este contaba con un plan de beneficios conocido como el Plan Obligatorio de Salud (POS) - actualmente Plan de Beneficios en Salud- el cual, era un listado de servicios de salud básicos a los cuales tenían derecho todas las personas que se encontraban afiliadas a 
este sistema; este plan de beneficios tenía como objetivo central brindar una protección integral a toda la población y fomentar la prevención y tratamiento de todas las enfermedades.

Es importante señalar que en esta lista no se mencionaba los procedimientos experimentales, lo que se convertía en un obstáculo para atender de forma integral todas las patologías, puesto que existen enfermedades que no tienen un tratamiento acreditado, por lo tanto, los pacientes tienen como única alternativa someterse a estos procedimientos que se encuentran en fase de experimentación.

Con el paso de los años y el significativo avance de la medicina, esta lista taxativa de beneficios POS se encontraba desactualizada, a pesar de que debía renovarse cada 2 años, lo anterior llevó a que los derechos fundamentales de los usuarios se vieran afectados y que el Estado adoptara diferentes medidas, como lo fue la implementación de la Ley Estatutaria de Salud, la Ley 1751 de 2015.

Para contextualizar lo anterior, en el año 2008 la Corte Constitucional a través de la sentencia T-760 abordó la necesidad de realmente adoptar medidas para eliminar incertidumbres relacionadas a los planes de beneficios, ya que había pasado un periodo de tiempo suficiente y razonable para verificar y actualizar condiciones en salud, esto en búsqueda de mejorar la prestación de servicios, pues la ausencia de dicha revisión integral daba origen a acciones de tutelas, donde el accionante buscaba resolver sus dudas acerca de lo que se encontraba incluido o excluido del POS. Por lo expuesto, se le ordenó a la Comisión Nacional de Regulación en Salud la actualización integral de los Planes Obligatorios de Salud (POS), estableciendo que se encuentra incluido y excluido de los planes de beneficios (Corte Constitucional, 2008).

La Ley 1751 de 2015 introdujo un cambio estructural trascendental en el sistema de salud, dado que eliminó el Plan Obligatorio de Salud y, en su lugar, implementó el Plan de Beneficios en Salud transformando la lista cerrada de servicios por un régimen de exclusión, en el cual se mencionan únicamente los tratamientos que no serán financiados con los recursos del Estado, en el que se resalta entre otros, los procedimientos experimentales.

Colombia como Estado constitucional, social y democrático de derecho, debe velar por una efectiva realización de los derechos fundamentales los cuales, como se ha observado en el presente texto, no son solo los que se encuentran en el Carta Política, sino también aquellos que los jueces reconocen en sus providencias, por lo que el derecho a "que sea intentado” tiene el carácter de fundamental. 
Este nuevo derecho reconocido recientemente guarda una estrecha relación con el derecho a la vida y la autonomía ya que el paciente adquiere un rol relevante, y, cuando este decide aceptar o rechazar un procedimiento experimental, está autodeterminándose y esta decisión debe ser respetada por toda la esfera social, por esta razón el Estado debe protegerlo y garantizarlo. En consecuencia de lo anterior, es claro que en este punto se presenta una tensión entre el derecho del paciente y la justicia social en salud dado que, por un lado está la voluntad de la persona, y, por el otro el derecho a la salud en condiciones dignas y bajo parámetros que no atenten contra la vida e integridad de la persona, es por esto que, como se mencionó precedentemente, se hace necesario regular este tema, con el objetivo de establecer algunos límites para su ejercicio, pues de no ser así, el paciente podría presentar un encarnizamiento con relación a estas alternativas experimentales y, simultáneamente, podría atentar contra sus demás derechos.

Así mismo, es necesario que se eliminen las barreras existentes entre el derecho a la salud y el procedimiento experimental, ya que si no se garantiza la realización de estos últimos, el Estado estaría interfiriendo en el plan de vida del paciente y, concomitante a esto, estaría transgrediendo la dignidad humana de este, sin embargo, se resalta que se da lugar a dicha transgresión, siempre y cuando, el paciente o su familia acepte el sometimiento al procedimiento experimental a través del consentimiento informado, pues por medio de este se comparte responsabilidades y se asumen riesgos.

Con el reconocimiento que hizo la Corte Constitucional frente al derecho fundamental a "que sea intentado" surge la necesidad de que el Estado colombiano lo reconozca plenamente como un derecho inviolable y le permita una aplicación directa, siendo necesaria una modificación en el régimen de exclusión, donde se eliminen los procedimientos experimentales y puedan estos, con la ayuda de la ciencia, ser utilizados en pro de la sociedad eliminando así una de las barreras del sistema de salud.

\section{Mecanismos Idóneos para Hacer Efectivo el Derecho a "Que Sea Intentado"}

Para comenzar, se requiere señalar que según el artículo 5 de la Constitución Política, el Estado reconoce, sin discriminación alguna, la primacía de los derechos inalienables de la persona, es decir, los derechos fundamentales, sin embargo, para darles una mayor protección se determinó a través del artículo 241 del mismo texto supremo, que la Corte Constitucional es la entidad judicial encargada de velar por su integridad y supremacía. 
En consideración de lo anterior, surgen los mecanismos de protección de derechos, los cuales nacen con el propósito de proteger derechos vulnerados o en situación de amenaza, puesto que no basta con un simple reconocimiento de estos, sino un debido control para garantizar su desarrollo efectivo.

Se hace necesario profundizar en la acción de tutela, por ser este el medio idóneo para reclamar el derecho fundamental innominado a "que sea intentado".

El artículo 86 de la Constitución Política establece que;

Toda persona tendrá acción de tutela para reclamar ante los jueces, en todo momento y lugar, mediante un procedimiento preferente y sumario, por sí misma o por quien actúe a su nombre, la protección inmediata de sus derechos constitucionales fundamentales, cuando quiera que éstos resulten vulnerados o amenazados por la acción o la omisión de cualquier autoridad pública $[. .$.

Así mismo, para darle un mayor sustento a lo establecido, la acción de tutela se encuentra reglamentado por el Decreto 2591 del 19 de noviembre de 1991, en el cual se establecen sus disposiciones generales y procedimiento, competencia, tutela contra los particulares, la relación existente entre la tutela y el defensor del pueblo y por último las sanciones que se pueden presentar en caso de que la autoridad que se encuentre vulnerando el derecho fundamental lo continúe haciendo a pesar de existir un fallo favorable.

Con lo precedente, se evidencia que la acción de tutela es un medio ágil y eficaz, orientado a ejercer un control constitucional frente a las acciones $\mathrm{u}$ omisiones presentadas por las autoridades públicas, y de manera esporádica, por un particular. No obstante, se puede generar una tensión, puesto si el juez accede a las pretensiones de la mayoría de los ciudadanos, se puede generar una afectación económica insostenible para el Estado, lo anterior, ha sido producto de un mal funcionamiento del Sistema General de Seguridad Social en Salud, por esta razón el juez debe valorar que el principio de sostenibilidad fiscal no puede ser tomado como criterio para negar el derecho a la salud.

\section{Conclusiones}

Si bien el derecho fundamental a "que sea intentado" no se encuentra expresamente en la Constitución Política de 1991, este ha sido desarrollado en 
relación con el artículo 94 del mismo precepto, dejando en evidencia que los derechos fundamentales en Colombia no se constituyen en una lista cerrada, ya que siempre se está en búsqueda de garantizar la dignidad humana; es por ello que, como ya lo reseñaba la sentencia T-406 de 1992 con el advenimiento del Estado Social de Derecho, "los derechos son aquello que los jueces dicen a través de las sentencias de tutela”. (Corte Constitucional, 1992, p. 14)

El mecanismo de protección de derechos idóneo para amparar el derecho a "que sea intentado" es la acción de tutela, puesto que se está ante un derecho fundamental innominado que ha sido reconocido a través de la sentencia T-057 de 2015, la cual es considerada una sentencia hito; pues si bien este es el único caso que se ha presentado en Colombia, puede servir de base para trazar una posible línea jurisprudencial en casos análogos que se puedan presentar frente al derecho a "que sea intentado" o a intentar procedimientos experimentales en determinadas circunstancias.

Para poder lograr una mayor eficacia en el derecho fundamental a "que sea intentado", se hace necesario una reforma legislativa en materia de salud donde se eliminen las barreras en cuanto al acceso de los procedimientos experimentales, toda vez que la actual Ley Estatutaria de Salud contraría un derecho fundamental que tiene conexidad con la vida y la dignidad humana; para esto, el Estado deberá aumentar el Presupuesto General de la Nación en la partida para salud.

Si bien el derecho a "que sea intentado" busca garantizar una mejor calidad de vida en condiciones dignas para el paciente que padece una enfermedad grave, también se convierte en un medio de esperanza para la familia. De hecho, hay debates que plantean lo problemático del reconocimiento de este derecho pues podría llevar a la "distanasia" o encarnizamiento terapéutico por parte de familiares, aún en contra de la dignidad humana del paciente. Es menester resaltar que la razón de ser de este nuevo derecho es permitir que la persona tenga una calidad de vida digna, lo que en palabras de Martha Nussbaum se traduce en: "poder vivir hasta el término de una vida humana de una duración normal; no morir de forma prematura o antes de que la propia vida se vea tan reducida que no merezca la pena vivirla" (Guichot Reina, 2015, p. 6), en ese orden de ideas, el derecho a "que sea intentado" no es un derecho absoluto, dado que tiene como límite la vida en condiciones dignas, es decir, que la persona pueda desarrollarse plenamente en el ámbito corporal y espiritual y que no deba ver afectada su integridad por la intención de someterse a un tratamiento experimental. Lo anterior abre la posibilidad a 
nuevas investigaciones al respecto, que más allá de describir el alcance de este nuevo derecho, problematicen su contenido y la pugna que entre el principio a la dignidad humana puede surgir en cabeza de titulares diferentes (familiares y pacientes).

Frente al desconocimiento de este nuevo derecho emergente, es imprescindible realizar capacitaciones tanto a los Comités Médicos en la red de hospitales, como a la ciudadanía en general, con el objeto de que primeramente la sociedad conozca este nuevo derecho fundamental y lo pueda solicitar en las Entidades Prestadores de Salud, y seguidamente, que estas últimas tengan estructurada una ruta de atención efectiva en caso de que un paciente que presente una enfermedad grave solicite someterse a un procedimiento experimental.

Así mismo, es necesario que se cree un Comité interdisciplinario encargado de brindar el acompañamiento requerido tanto al paciente que se somete al procedimiento experimental, como a la familia que es parte fundamental en el proceso.

Por otro lado, es importante señalar que los procedimientos experimentales se convierten en una gran oportunidad para el progreso de la humanidad, lo cual permitirá encontrar posibles soluciones a graves enfermedades que afectan a la sociedad.

Por último, puede decirse que a pesar de que el Congreso no ha legislado sobre el derecho a "que sea intentado" por ser este un tema que suscita un debate religioso, moral y legal, es imperativo que se legisle acerca de este derecho, por ser éste de carácter fundamental, con esto se evitaría ampararlo vía acción de tutela y de esta manera se garantizaría su efectiva aplicación, evitando así mismo un desgaste judicial.

\section{Referencias}

Aguiló Regla, J. (2007). Positivismo y postpositivismo dos paradigmas jurídicos en pocas palabras. Alicante: Universidad de Alicante

Amaya. J. A. (2015). Control de Constitucionalidad. Bogotá: Astrea

Chinchilla Herrera, T. E. (2009). ¿Qué son y cuáles son los derechos fundamentales? Bogotá: Temis

Colombia. Corte Constitucional (agosto13 de 1992). Sentencia T-491. Recuperado de: https://www.corteconstitucional.gov.co/relatoria/1992/T-491-92.htm 
Colombia. Corte Constitucional (junio 5 de 1992). Sentencia T-406. Recuperado de: https://www.corteconstitucional.gov.co/relatoria/1992/T-406-92.htm

Colombia. Corte Constitucional (mayo 12 de 1999), Sentencia SU-337. Recuperado de: https://www.corteconstitucional.gov.co/relatoria/1999/ su337-99.htm\#: :text=SU337\%2D99\%20Corte\%20Constitucional\%20de\%20 Colombia\&text=Los\%20procesos\%20judiciales\%20deben\%20ser,distintos\%20 jueces\%20en\%20la\%20materia.

Colombia. Corte Constitucional (marzo 17 de 2003), Sentencia T-227. Recuperado de: $\quad$ https://www.corteconstitucional.gov.co/relatoria/2003/t-227-03. htm\#: : text = T\%2D 227\%2D03\%20 Corte\% 20 Constitucional\% 20 de $\% 20$ Colombia \& text $=$ La 20 Corte $\% 20$ Constitucional $\% 20$ ha $\% 20$ fijado,fundamentales\%20por\%20v\%C3\%ADa\%20de\%20tutela.\&text=Se\%20 trata\%20de\%20la\%20selecci\%C3\%B3n, desarrollo\%20jurisprudencial\%20de\%20 la\%20Constituci\%C3\%B3n\%E2\%80\%9D.

Colombia. Corte Constitucional (mayo 10 de 2006), Sentencia C-355. Recuperado de: https://www.corteconstitucional.gov.co/relatoria/2006/c-355-06.htm

Colombia. Corte Constitucional (septiembre 11 de 2008), Sentencia T-881. Recuperado de: https://www.corteconstitucional.gov.co/relatoria/2008/T-881-08.htm

Colombia. Corte Constitucional (mayo 15 de 2008), Sentencia C-483. Recuperado de: https://www.corteconstitucional.gov.co/relatoria/2008/C-483-08.htm

Colombia. Corte Constitucional (febrero 29 de 2008), Sentencia T-216. Recuperado de: https://www.corteconstitucional.gov.co/relatoria/2008/T-216-08.htm

Colombia. Corte Constitucional (mayo 23 de 2013), Sentencia T-310. Recuperado de: https://www.corteconstitucional.gov.co/RELATORIA/2013/T-310-13.htm

Colombia. Corte Constitucional (abril 2 de 2013), Sentencia T-180. Recuperado de: https://www.corteconstitucional.gov.co/RELATORIA/2013/T-180-13.htm

Colombia. Corte Constitucional (diciembre 15 de 2015), Sentencia T-1330. Recuperado de: https://www.corteconstitucional.gov.co/relatoria/2005/T-1330-05.htm

Colombia. Corte Constitucional (febrero 12 de 2015), Sentencia T-057. Recuperado de: https://www.corteconstitucional.gov.co/relatoria/2015/t-057-15.htm

Defensoría del Pueblo Colombia. (s.f.). Solicitud de mecanismos de protección de Derechos Humanos. Recuperado el 18 de abril de 2019 de: http://www.defensoria. gov.co/es/public/atencionciudadanoa/1471/Solicitud-de-mecanismos-deprotecci\%C3\%83\%C2\%B3n-de-Derechos-Humanos.htm

DelGrosso, D. (2017). Fighting for your life in America: A study of "Right to Try" Laws throughout the Country. Nueva York: St. John's University

Echavarría, J. L. (2013). De la naturaleza jurídica del derecho a la salud en Colombia. Colombia: Superintendencia Nacional de Salud

Richardson, E. (6 de marzo de 2017). Federal right-to-try legislation undermines patient protection and drug testing. Recuperado el 23 de enero de 2019 de: https://www. statnews.com/2017/03/06/right-to-try-law-fda/

Escobar-Picasso, E., Escobar Cosme, A. (2010). Principales corrientes filosóficas en bioética México D.F.:Hospital Infantil de México. Recuperado de: http://www.scielo. org.mx/pdf/bmim/v67n3/v67n3a3.pdf

Guichot Reina, V. (2015). "El enfoque de las capacidades" de Martha Nussbaum y sus consecuencias educativas: hacía una pedagogía socrática y pluralista. Salamanca: Universidad de Salamanca. Recuperado de: https://gredos.usal.es/bitstream/ 
handle/10366/127651/El_\%ABenfoque_de_las_capacidades\%BB_de_Marth.pdf;jse ssionid $=$ FC8693345C087C947A68B805F3BEA6EC? sequence $=1$

Howard, J. (2018). What you need to know about right-to-try legislation. Recuperado el 15 de abril de 2019 de: https://edition.cnn.com/2018/03/22/health/federal-rightto-try-explainer/index.html

Jiménez, W. G.; Angulo, L. L.; Angulo, Y. P.; Gómez, M. L.; Rey, L. J.; Solano, L. T.; Urquijo, Y. C. (2016). Ley Estatutaria: ¿avance Hacia La garantía Del Derecho Fundamental a La Salud? Revista Colombia, Volumen 31, (p.81 - 90).

López Medina, D. E. (2015). El derecho de los jueces. Bogotá, Colombia: Legis

Martínez, C. A. (2016). La génesis de la Constitución Política de Colombia de 1991 a la luz de la discusión sobre el Mito Político. Bogotá, Colombia: Universidad Rosario

Mazo Álvarez, H. M. (2014). El bioderecho: La respuesta jurídica a los problemas que plantea la bioética. Medellín, Colombia: Corporación Universitaria Remington

Monroy Cabra, M. G. (2015). Introducción al derecho. Bogotá, Colombia: Temis

March, R. (11 de julio de 2017). Right To Try laws offer hope for the terminally ill. Recuperado el 30 de enero de 2019 de: https://thehill.com/opinion/ healthcare/359148-right-to-try-laws-offer-hope-for-the-terminally-ill

Right to Try ORG. (2018). What is the right to try Recuperado el 23 de enero de 2019 de: http://righttotry.org/about-right-to-try/

Nieto Sánchez A.L. (s.f). La Corte Constitucional Colombiana como legislador en sentido positivo. Bogotá, Colombia: Universidad Católica de Colombia 